\title{
Stacking of ZnSe/ZnCdSe Multi-Quantum Wells on GaAs (100) by Epitaxial Lift-Off
}

\author{
N.M. ELDOSE, ${ }^{1,2}$ J. ZHU, ${ }^{1}$ N. MAVRIDI, ${ }^{1}$ KEVIN PRIOR, ${ }^{1}$ and R.T. MOUG ${ }^{1}$ \\ 1.-School of Engineering and Physical Sciences, Heriot-Watt University, Edinburgh EH14 4AS, \\ UK. 2.-e-mail: nm20@hw.ac.uk
}

Here we present stacking of GaAs/ZnSe/ZnCdSe single-quantum well (QW) structures using epitaxial lift-off (ELO). Molecular beam epitaxy (MBE)grown II-VI QW structure was lifted using our standard ELO technique. The QW structures were transferred onto glass plates and then subsequent layers stacked on top of each other to form a triple-QW structure. This was compared to an MBE-grown multiple-QW (MQW) structure of similar design. Lowtemperature $(77 \mathrm{~K})$ photoluminescence $(\mathrm{PL})$ spectroscopy was used to compare the two structures and showed no obvious degradation of the ELO stacked layer. It was observed that by stacking the single QW layer on itself we could increase the PL emission intensity beyond that of the grown MQW structure while maintaining narrow line width.

Key words: Stacking, ELO, MBE, PL, II-VI, MQW

\section{INTRODUCTION}

Epitaxial lift-off (ELO) is a post-growth process used to exfoliate layers from their growth substrate allowing transfer to different functional surfaces. ${ }^{1,2}$ ELO was originally demonstrated in III-V materials by Yablonovitch ${ }^{3}$ and extended to II-VI materials by our group at Heriot Watt using MgS as our sacrificial layer. ${ }^{2,4} \mathrm{MgS}$ is easily soluble in $\mathrm{HCl}$ with a high etch rate compared to other II-VI materials, $\sim$ 10,000:1. This allows for quick and consistent exfoliation of the II-VI layers. Our group hse previously shown that the lifted layers can be transferred to a range of alternative substrates including $\mathrm{BO}_{2} \mathrm{Si}_{3}$ glass, distributed Bragg reflectors (DBR's) and lithium niobate surfaces for a range of functional applications. ${ }^{4}$ We recently extended IIVI ELO to the three main commercially available III-V substrates, still using MgS as the sacrificial layer. ${ }^{5,6}$

Studies recently have focused on combining semiconductor structures using novel post-growth processing such as direct wafer bonding to overcome some inherent growth or material limitations. ${ }^{7}$ This

(Received December 26, 2017; accepted April 20, 2018;

published online May 4, 2018) process showed that two different semiconductor layers can be combined together in one structure. In this paper, we aim to show that II-VI ELO can provide a more generalized fabrication technique. Herein we present that by exfoliating a single quantum well (QW) of $\mathrm{ZnSe} / \mathrm{Zn}_{x} \mathrm{Cd}_{1-x}$ Se from its substrate, and stacking it on top of itself, we can manufacture a structure of comparable quality to that of a molecular beam epitaxy (MBE)-grown multi-QW (MQW) structure of the same design. Photoluminescence (PL) spectroscopy is used to compare the optical emission from the stacked QW layer and an MBE-MQW grown structure. By combining this stacking process with other ELO functionality, we hope to highlight that ELO can be used to generate systems and structures not currently feasible by standard growth and fabrication techniques.

\section{GROWTH}

Samples were grown on $n^{+}$GaAs (100) substrates using a Vacuum Generators V80H MBE system. Sources of $6 \mathrm{~N}$ purity elements of $\mathrm{Zn}, \mathrm{Cd}$, Se, $\mathrm{Mg}$, together with compound source of $\mathrm{ZnS}$ were used with the following cell temperatures: $\mathrm{Zn} 290^{\circ} \mathrm{C}$, Se $183^{\circ} \mathrm{C}, \mathrm{Mg} 350^{\circ} \mathrm{C}, \mathrm{Cd} 195^{\circ} \mathrm{C}$ and $\mathrm{ZnS} 865^{\circ} \mathrm{C}$. Nominal flux ratios were set to be 1:2 and 1:2:2 for the 
ZnSe and $\mathrm{Zn}_{(0.79)} \mathrm{Cd}_{(0.21)}$ Se, respectively. The $\mathrm{ZnS}$ source was cooled by a liquid nitrogen cold shutter to prevent substrate contamination during the substrate de-oxidation process. The growth temperature was set at $240^{\circ} \mathrm{C}$ for all samples. Two samples were grown: the first was a single-QW $\mathrm{GaAs}_{(100))} / \mathrm{ZnSe}_{(10 \mathrm{~nm})} / \mathrm{MgS}_{(6 \mathrm{~nm})} /$ $\mathrm{ZnSe}_{(70 \mathrm{~nm})} / \mathrm{ZnCdSe}_{(10 \mathrm{~nm})} / \mathrm{ZnSe}_{(70 \mathrm{~nm})}$, and the second was a MQW of the same structure with $3 \times \mathrm{ZnSe} /$ ZnCdSe well repeats. Using lattice constants for $\mathrm{GaAs}, \mathrm{ZnSe}$, and $\mathrm{MgS}$ it is seen that there is a $0.2 \%$ (compressive) mismatch for $\mathrm{ZnSe}$ to GaAs and a $0.6 \%$ (tensile) mismatch for MgS. This provides a small strain balance effect between the lift-off layer and the subsequent QW and has been shown in previous experiments to qualitatively improve the PL. As such, the MgS layer was also added into the MQW sample for a more direct comparison.

The growth parameters remained the same for both samples and were grown under the same flux ratio, growth temperature, and on the same day to allow for a direct comparison between them. The thickness of the layers were previously calibrated using a combination of a DEKTAK thickness profiler and x-ray interference (XRI) characterisation and yielded growth rates of $0.55 \AA / \mathrm{s}$ and $0.88 \AA / \mathrm{s}$ for the $\mathrm{ZnSe}$ and $\mathrm{ZnCdSe}$, respectively. XRI was also used to determine the QW composition as $\mathrm{Zn}_{(0.79)} \mathrm{Cd}_{(0.21)}$ Se. $^{5}$ An initial $\mathrm{ZnSe}$ buffer layer is used to protect the substrate from $\mathrm{S}$ contamination and provides the best surface for the growth of MgS sacrificial layer. ${ }^{4,8}$ The normal ZnSe $2 \times 1$ and MgS $\mathrm{C}(2 \times 2)$ reflection high-energy electron diffraction (RHEED) patterns ${ }^{9}$ were observed during both layer growths. At this stage the samples were characterized by photoluminescence (PL) spectroscopy using a 375-nm GaN diode laser focussed onto the sample and emission collected by a chargecoupled device (CCD) spectrometer. PL emission for single and triple QW was measured at room temperature (RT) and low temperature (LT) at $77 \mathrm{~K}$. The results are discussed in "Results" section.

\section{ELO AND SAMPLE STACKING}

For this experiment, a modified version of our ELO procedure was used. Samples were then cleaved into $5 \times 5-\mathrm{mm}$ pieces and then adhered onto the polymer film. The samples were placed face up in a solution of $30 \% \mathrm{HCl}$ at room temperature. Once the etching is completed, the substrate falls down to the bottom of the container, leaving the lifted layer on the polymer film on the surface. Commercially available electrostatic polymers, commonly used to protect glass phone screens, were used in place of our standard wax carrier method demonstrated previously. ${ }^{10}$ This film gives the sample structure, provides an upward force to lift the layer during etching and keeps the cleaved edges clear to allow etching to penetrate. The lifted layer is carefully taken from the solution and rinsed in deionised water while still attached to the polymer film and the newly exposed semiconductor surface coated with clear wax. The polymer film is then removed, leaving the sample encapsulated in wax, and the sample transferred to the glass slide while retaining structural rigidity.

The layer was transferred to a glass plate using water as an adhesive medium. The water was evaporated gradually by keeping a small pressure on top the wax for 6-12 h. Wax was removed by dipping in acetone for $15 \mathrm{~min}$. These steps were repeated for each lifted layer and layers were then stacked on top each other.

The thickness of each ELO layer on the glass plate was measured using a DEKTAK thickness profiler and shown in Fig. 1. The linear increase in the thickness of each layer has an error of $\pm 10 \%$ and, as such, is not overly accurate; however, this demonstrates that the layers have been stacked successfully. The comparison of triple-stacked single $\mathrm{QW}$ on a glass plate and triple $\mathrm{QW}$ grown on GaAs substrate shows roughly the same thickness within an error of $\pm 10 \%$. The layers were stacked off-axis to each other to create steps for the thickness measurement. This is summarised in Table I, "Results" section.

\section{RESULTS}

To examine the quality of the stacked layer, PL measurements of both single and triple QWs on GaAs substrates were taken and compared to the triple-stacked single QW on the glass plate. All samples were measured at $77 \mathrm{~K}$ with the same excitation power $(25 \mathrm{~mW})$ and integration time

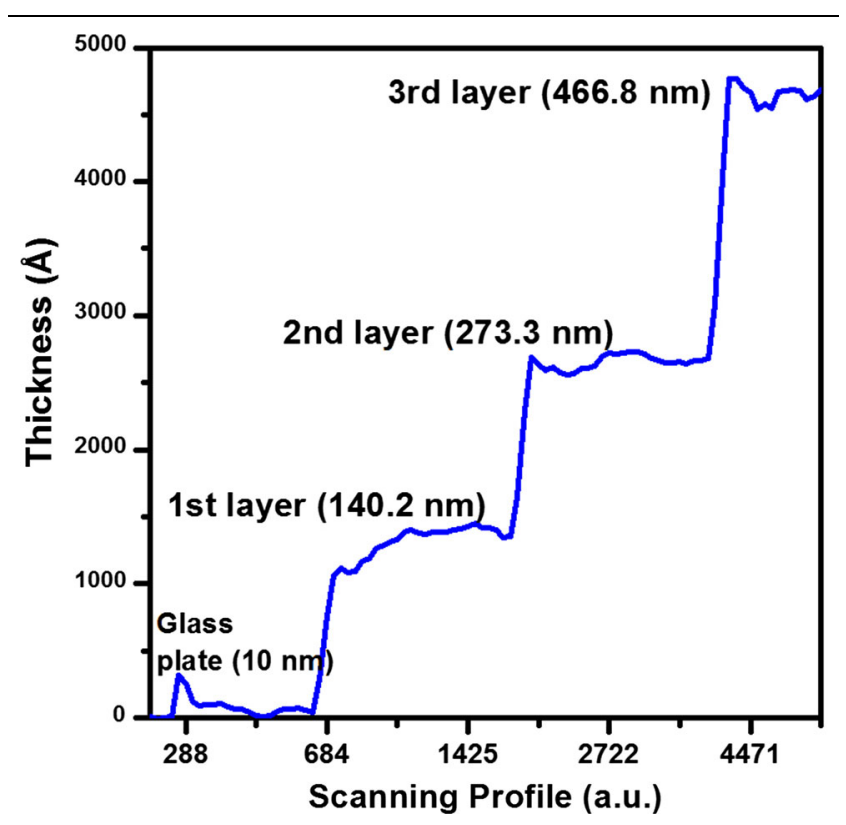

Fig. 1. Thickness measurement taken using a DEKTAK thickness profiler. The blue line indicates total structural thickness after each additional layer. The stacked layers were positioned off-axis to each other to specifically enable measurement. 
Table I. The change in the peak position, FWHM and thickness of single QW, triple $Q W$ and triple-stacked single QW

\begin{tabular}{|c|c|c|c|c|c|}
\hline \multirow[b]{2}{*}{$\mathbf{Q W}$} & \multicolumn{2}{|c|}{ FWHM (meV) } & \multicolumn{2}{|c|}{ Energy (eV) } & \multirow[b]{2}{*}{ Thickness (nm) } \\
\hline & $77 \mathrm{~K}$ & RT & $77 \mathrm{~K}$ & RT & \\
\hline $1 \times$ & 33.64 & 56.36 & 2.456 & 2.39 & 150 \\
\hline $3 \times$ & 33.27 & 59.62 & 2.458 & 2.385 & 450 \\
\hline $3 \times$ Stack & 35.01 & 81.35 & 2.458 & 2.387 & 465 \\
\hline
\end{tabular}

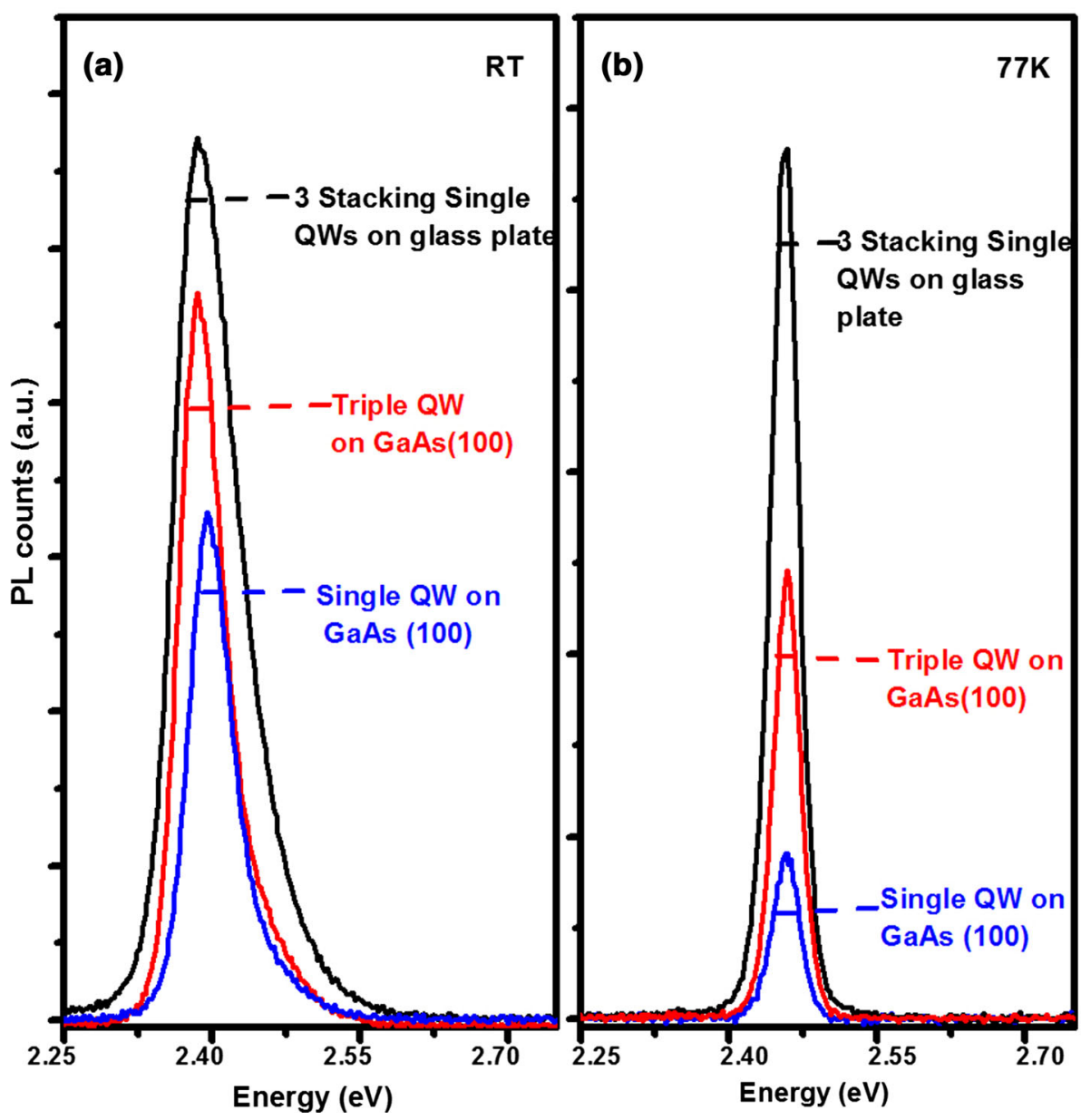

Fig. 2. PL spectra of single, triple $\mathrm{QW}$ and triple-stacked single $\mathrm{QW}$ at (a) $77 \mathrm{~K}$ and (b) RT. All measurements were taken under same conditions so a relative intensity comparison can be made.

(10 s), and at RT with an excitation power of $65 \mathrm{~mW}$ and an integration time of $10 \mathrm{~s}$. The resultant spectra is shown in Fig. 2. Table I shows the values of full width at half maximum (FWHM), emission energy and sample thickness measured for all samples. It can be seen from the results that there is negligible shift in PL peak position $(\sim 2 \mathrm{meV})$ of the stacked layer compared with that of the as-grown structure. This shows there is little strain induced during the ELO and stacking procedure. The $\mathrm{ZnSe}$ peak at $2.78 \mathrm{eV}$ is not visible in these spectra as the $\mathrm{QW}$ emission dominates, and to allow for comparison between samples we required a laser power low enough not to saturate our CCD detector. This reduced the ZnSe peak such that it can't be seen in these spectra. What is of great interest is the large increase in PL emission intensity of the stacked well. The intensity of the triple-stacked layer on glass is $\sim 200 \%$ larger compared to the triple $\mathrm{QW}$ grown on GaAs, this was seen to be true for both LT and RT measurements. 


\section{DISCUSSION}

There is a slight increase in the FWHM between the triple well and the stacked well at both $77 \mathrm{~K}$ and $\mathrm{RT}$. We suspect this is due to the surface roughness of the glass plate compared with the GaAs substrate. As the layer is adhered to the glass, we propose that the layer adopts the profile of the glass and generates pinch points in the layer; this in turn generates a variation in the $\mathrm{QW}$ emission and broadens the FWHM. The root mean square (RMS) of the glass plate was determined to be $\sim 11 \mathrm{~nm}$ compared to $\sim 0.4 \mathrm{~nm}$ for a bare GaAs wafer. This could easy be resolved with a higher polish of glass. It would advantageous at this stage to measure the RMS of the final stacked layer to investigate how this roughness propagates through successive layers and this will be a topic of future work. The increase in the PL emission intensity of the -triple-stacked layer compared to its as grown comparison is of great interest and completely unexpected. We suspect that non-radiative recombination effects, due to defects, increases in the grown layer once it has fully relaxed and limits the emission intensity. The single-well structure is below the critical thickness for relaxation for $\mathrm{ZnSe}$ $(\sim 140 \mathrm{~nm})$ and, therefore, the radiative recombination rate for each stacked layer remains the same. This provides an additive effect to the emission intensity for each additional layer, unhindered by defect generation. Therefore, in theory, the PL intensity emission of these stacked structures can be increased further by stacking more layers, limited only by absorption effects within the structure. In the future, we will study the limit of the PL intensity emission by stacking more layers.

More structural characterisation is required for this technique and in the future, we plan to attempt to obtain some x-ray diffraction (XRD) measurements of the stacked layer. In this study, as the layers were stacked on glass this was not feasible, but the process can be repeated on GaAs to demonstrate the structural integrity of each stacked layer. Alternatively, ellipsometry can be used to examine the layers optically. This will also give us more accurate thickness profiles and can be used to quantify surface roughness. This will also be the focus of future work.

During this study, a parallel investigation was undertaken to quantify the adhesion properties of the stacked layers. Preliminary results indicated that these layers are chemically bonded to each other and, once adhered, are fairly robust. This paper is currently submitted for review. In our study, the samples were routinely temperaturecycled from LT to RT for each subsequently stacked layer. We did not display these results in this paper for clarity reasons, but consistent PL was obtained for multiple layers and over various areas on the sample.
One area we hope to investigate further in the future is the electrical properties of the stacked layers. As these appear to be chemically bonded, there is the possibility that charge can flow between layers and, therefore, some electrically driven devices could be fabricated. Using II-VI material as a surface passivation layer is one area we hope to explore in the future and this will provide us with the first set of current-voltage measurements for the lifted layers.

\section{CONCLUSION}

We have demonstrated for the first time the stacking of multiple ELO layers whilst maintaining the structural and optical quality. It was seen that the stacked structure provides large PL intensities compared to the grown structures on substrates. It has also been shown that PL peak position does not shift after lifting off the epitaxial layer at RT and the QW emission is much higher in the stacked layers than in the QW structures grown on substrates. The stacked layers on glass plates undergo many temperature cycles during $77 \mathrm{~K}$ to $\mathrm{RT}$, after which it still has strong PL emission. The stacking of the lift-off process demonstrated that it can make designs which cannot be grown in normal growth chambers.

\section{OPEN ACCESS}

This article is distributed under the terms of the Creative Commons Attribution 4.0 International License (http://creativecommons.org/licenses/by/4.0/), which permits unrestricted use, distribution, and reproduction in any medium, provided you give appropriate credit to the original author(s) and the source, provide a link to the Creative Commons license, and indicate if changes were made.

\section{REFERENCES}

1. P. Demeester, I. Pollentier, P. Dobbelaere, C. Brys, and P.V. Daele, Semicond. Sci. Technol. 8, 1124 (1993).

2. M. Konagai, M. Sugimoto, and T. Takahashi, J. Cryst. Growth 45, 277 (1978).

3. E. Yablonovitch, T. Gmitter, J.P. Harbison, and R. Bhat, Appl. Phys. Lett. 51, 2222 (1987).

4. R.J. Warburton, C. Bradford, A. Currran, A. Balocchi, B.C. Cavenett, and K.A. Prior, J. Cryst. Growth 278, 325 (2005).

5. A. Rajan, I.A. Davidson, R.T. Moug, and K.A. Prior, J. Appl. Phys. 243510, 114 (2013).

6. A. Rajan, R.T. Moug, and K.A. Prior, Semicond. Sci. Technol. 29, 025006 (2014)

7. H. Moriceau, F. Rieutord, F. Fournel, Y. Le Tiec, L. Di Cioccio, C. Morales, A.M. Charvet, and C. Deguet, Adv. Nat. Sci. Nanosci. Nanotechnol. 043004, 11 (2010).

8. C. Bradford, C.B. O’Donnell, B. Urbaszek, A. Balocchi, C. Morhain, K.A. Prior, and B.C. Cavenett, Appl. Phys. Lett. 76,26 (2000)

9. R.T. Moug, C. Bradford, D. Thuau, A. Curran, R.J. Warburton, and K.A. Prior, J. Korean Phys. Soc. 53, 3004 (2008).

10. A. Balocchi, A. Curran, T.C.M. Graham, C. Bradford, K.A. Prior, and R.J. Warburton, Appl. Phys. Lett. 86, 011915 (2005). 